\title{
Bürgernahes Regieren
}

\section{Lässt sich politische Beteiligung durch E-Participation Anwendungen verbessern?}

\author{
Daniel Veit, Nils Parasie, Florian Schoppé \\ Dieter Schwarz Stiftungslehrstubl für ABWL, \\ E-Business und E-Government, \\ Universität Mannheim, \\ Schloss, 68131 Mannheim \\ \{veit|parasie|schoppe\}@bwl.uni-mannheim.de
}

\section{Einleitung}

„Hi everybody“ - Mit diesen Worten richtete sich der Präsident der Vereinigten Staaten, Barack Obama, zum ersten Mal nach seiner Wahl per Online-Videobotschaft direkt an seine Anhänger, ohne den Weg über die traditionellen Medien zu gehen (Moorstedt 2009, S. 15). Dieses Beispiel zeigt, dass eine neue Kultur der Kommunikation mit dem Bürger in den politischen Diskurs Einzug hält. Das Internet spielte eine davor nie dagewesene Rolle im US-amerikanischen Wahlkampf 2008 (Feldmer 2008, S. 21).

In diesem Beitrag wird anhand einer quantitativen empirischen Studie untersucht, inwiefern, durch die Bereitstellung elektronischer Beteiligungsmöglichkeiten, eine stärkere Teilnahme der Bürger an politischen Prozessen erreicht werden kann. Dazu stützt sich die Arbeit auf Literatur zur Akzeptanz von Technologien. Die Ergebnisse sollen dazu beitragen, Handlungsempfehlungen zu einer erfolgreichen Umsetzung von E-Participation Anwendungen im Rahmen der politischen Meinungsbildung zu geben. In Abschnitt 2 wird zunächst der aktuelle Forschungsstand zur elektronischen Beteiligung wiedergegeben. In Abschnitt 3 wird das verwendete Forschungsmodell entwickelt, welches anschließend in Abschnitt 4 einem empirischen Test unterzogen wird. Die Ergebnisse werden in Abschnitt 5 diskutiert. Abschnitt 6 fasst die Arbeit zusammen. 


\section{Forschungsstand}

Verglichen mit anderen Disziplinen ist das Forschungsgebiet E-Participation ein relativ neues und in der Entwicklung stehendes Wissenschaftsfeld (Macintosh 2008, S. 85). Maßgebliche Forschungsvorhaben in diesem Bereich werden seit dem Aufkommen des Internets in den 1990er Jahren verfolgt (Parvez 2008, S. 21).

E-Democracy als Teilgebiet von E-Government kann definiert werden als ,[...] Einsatz moderner Informations- und Kommunikationstechnologien für Bürgerinitiativen, Parteien, Politiker und Wahlkämpfe bis hin zur Durchführung von Wahlen und Volksabstimmungen [...]" (Lucke und Reinermann 2000, S. 5). Dabei steht die Verbesserung und Intensivierung der politischen und demokratischen Kommunikation zwischen Repräsentant und Bürger mit Hilfe neuer Technologien im Vordergrund (Jansen et al. 2006, S. 5). E-Participation wird dabei als Teilgebiet von E-Democracy verstanden. Die im Jahr 2001 verfasste OECD Definition, die in der Literatur seither vielfach verwendet wird (Macintosh 2008, S. 89), beschreibt E-Participation durch drei Funktionen (Caddy und Vergez 2001, S. 21):

1. Informationsbereitstellung (einseitiger Informationsfluss): Bei dieser Funktion handelt es sich um eine einseitige Beziehung, durch die der Staat als Produzent von Informationen auftritt, z. B. durch die Bereitstellung von Informationen zu aktuellen politischen Diskussionen über öffentlich zugängliche Dokumente und Webseiten.

2. Konsultation (zweiseitiger Informationsfluss): Hierbei handelt es sich um eine beiderseitige Beziehung, bei der vom Staat Informationen bereitgestellt werden, auf die eine Rückmeldung durch die Bürger erfolgt. Auch hier geht die Initiative vom Staat aus, z. B. in Form von Meinungsumfragen.

3. Aktive Partizipation (Partnerschaft zwischen Bürger und Regierung): Diese Funktion umfasst die Möglichkeit, dass Bürger eigene Vorschläge zu politischen Entscheidungen einbringen können. Dabei steht die aktive Unterstützung engagierter Bürger im Vordergrund.

Nach anfänglich technikorientierter Betrachtung, stehen seit den 1990er Jahren auch soziale Aspekte von E-Participation Angeboten im Fokus (Sclove 1995, S. 18). Dennoch mangelt es an empirisch fundierten, repräsentativen Studien, die eine Aussage über Erfolgsfaktoren solcher Angebote möglich machen (Albrecht et al. 2008, S. 6).

Die in den vergangenen Jahren aufgekommenen sozialen Internetanwendungen könnten E-Participation Möglichkeiten maßgeblich unterstützen. Unter dem Begriff Web 2.0 wird eine veränderte Nutzung und Wahrnehmung des Internets verstanden, bei der sich der Internetnutzer vom Konsumenten zum Produzenten von Inhalten wandelt (O’Reilly 2005, S. 2). Diese erweiterten Möglichkeiten der individuellen politischen Kommunikation werden in jüngeren Arbeiten als Katalysator der Verbreitung von politischer Partizipation angesehen (Stern 2008, S. 179). Bräuer et al. (2008, S. 204) stellen fest, dass Web 2.0-Nutzer häufiger Seiten von 
Politikern besuchen, politische Themen diskutieren und direkten Kontakt mit Personen in einem politischen Amt haben als Personen, die nicht im Web 2.0 aktiv sind.

Zur Akzeptanz von E-Participation in Europa wurde vor allem die Motivation zur Nutzung solcher Dienste durch die Bürger als unzureichend erforschte Variable identifiziert. Auf Basis der bisherigen Ergebnisse ließ sich jedoch kein Modell entwickeln, mit dem sich die Akzeptanz und damit der Erfolg von E-Participation Anwendungen vorhersagen lässt (Westholm und Wimmer 2007, S. 58).

Vor diesem Hintergrund werden in diesem Beitrag Einflussfaktoren auf die Akzeptanz von E-Participation Anwendungen untersucht. Untersuchungsgegenstand sind dabei Anwendungen, die der Kommunikationsbeziehung zwischen Repräsentant und Bevölkerung dienen. Als Grundlage dient das Technology Acceptance Model (Davis et al. 1989, S. 983), welches auf die spezifischen Charakteristika von E-Participation Anwendungen angepasst und erweitert wird.

\section{Modellentwicklung}

Das Technology Acceptance Model (TAM) gilt als robustes und etabliertes Modell zur Vorhersage von Nutzerakzeptanz innovativer Systeme (Venkatesh et al. 2003; S. 428; Jeyaraj et al. 2006, S. 14). In der von Davis et al. (1989, S. 983) veröffentlichten Ursprungsversion basiert das Modell auf der Theory of Reasoned Action (TRA) von Fishbein und Ajzen (1975) durch die die Verhaltensabsicht einer Person als wesentlicher Faktor für das tatsächliche Verhalten etabliert wurde. TAM ist eine Adaption von TRA und wurde speziell auf die Modellierung der Anwenderakzeptanz von Informations- und Kommunikationssystemen angepasst (Davis et al. 1989, S. 985).

Ziel von TAM ist es, die allgemeinen Determinanten der Akzeptanz von Computersystemen und Software für eine Vielzahl unterschiedlicher Systeme zu erklären. Das Model propagiert folgende Einflussfaktoren für die Nutzerakzeptanz neuer Informationssysteme (Davis et al. 1989, S. 985; Davis 1989, S. 320):

- Erwarteter Nutzen (EN): Subjektive Einschätzung bezüglich des zukünftigen

Nutzens, der durch die Verwendung einer spezifischen Software oder eines Systems erwartet wird.

- Wahrgenommene Einfachbeit (WE): Ausmaß zu dem das System nach Einschätzung des Anwenders ohne besondere Anstrengungen nutzbar ist.

Die durch das Modell postulierten Einflussfaktoren konnten seither in zahlreichen weiteren Studien bestätigt werden (Venkatesh et al. 2003, S. 428; Jeyaraj et al. 2006, S. 14). Die aus TAM abgeleiteten Hypothesen, die in Bezug auf E-Participation Anwendungen untersucht werden sollen, lauten:

H1: Einfachbeit der Nutzung einer E-Participation Anwendung wirkt sich positiv auf den erwarteten Nutzen der Anwendung aus. 
H1 $1_{b}$ Einfachbeit der Nutzung einer E-Participation Anwendung wirkt sich positiv auf die Nutzungsabsicht aus.

H2: Ein hoher erwarteter Nutzen einer E-Participation Anwendung wirkt sich positiv auf die Nutzungsabsicht aus.

Darüber hinaus ist es für Menschen im virtuellen Raum schwerer einzuschätzen, ob das Gegenüber ihnen potentiell schaden will oder wohlgesonnen ist, als bei einer realen Begegnung mit persönlicher Kommunikation (Friedmann et al. 2000, S. 39). Bei der Verwendung von E-Participation Anwendungen, bei der persönliche Meinungen und Überzeugungen preisgegeben werden, besteht eine besondere Herausforderung an die Vertraulichkeit der verwendeten elektronischen Systeme (Coleman und Gotze 2001, S. 5). Die Bedeutung von Vertrauen im Umgang mit elektronischen Systemen wird ferner durch das Urteil des Bundesverfassungsgerichtes vom 27.02.2008 unterstrichen, durch welches ein Grundrecht in die Integrität und Vertraulichkeit informationstechnischer Systeme garantiert wird ${ }^{1}$. Daher kann eine vertrauensvolle Umsetzung von E-Participation Anwendungen als wesentlicher Faktor für die Akzeptanz dieser Beteiligungsmöglichkeit angesehen werden (Dutton und Shepherd 2006, S. 433, Kubicek und Westholm 2005, S. 39).

Die Verbindung zwischen Vertrauen und TAM wurde in der Literatur bereits diskutiert (Wu und Chen 2005, S. 789; Carter und Bélanger 2005, S. 18). Pavlou (2003, S. 90) entwickelte ein um den Faktor Vertrauen erweitertes TAM und konnte einen starken positiven Einfluss des Faktors Vertrauen auf die Konstrukte erwarteter Nutzen und Nutzungsabsicht im Rahmen einer E-Commerce Studie feststellen.

Im Bereich E-Government wurde TAM von Carter und Bélanger (2005, S. 18) um das Konstrukt Vertrauen erweitert und anhand der Nutzung von BehördenWebseiten getestet. Dabei wurde ein signifikanter Einfluss der wahrgenommenen Vertrauenswürdigkeit auf die Nutzungsabsicht ermittelt. Um eine präzisere Aussage über den Einfluss von Vertrauen auf die Nutzung von E-Participation Anwendungen machen zu können, wird das Konstrukt Vertrauen auch in dieser Arbeit als Einflussfaktor untersucht.

$\mathrm{H}_{a}:$ Vertrauen in eine E-Participation Anwendung wirkt sich positiv auf den erwarteten Nutzen aus.

$\mathrm{H}_{6}$ : Vertrauen in eine E-Participation Anwendung wirkt sich positiv auf die Nutzungsabsicht aus.

Neben den demographischen Merkmalen, wie Alter, Geschlecht und Tätigkeit der Probanden wird die politische Motivation als weiterer Faktor erfasst. Dadurch soll das Erklärungspotential des Theoriemodells erweitert werden (Sun und Zhang 2006, S. 53). Hierbei liegt der Fokus auf dem Interesse an politischen Themen und der Frage inwieweit der Proband die aktuellen politischen Geschehnisse verfolgt. Die Hypothese, die in diesem Zusammenhang untersucht werden soll, unterstellt, dass

${ }^{1}$ BVerfG, Urteil vom 27.2.2008, Aktennotiz 1 BvR 370/07 - 1 BvR 595/07 
E-Participation Anwendungen mit einem hohen erwarteten Nutzen überproportional stark von Bürgern mit einer hohen politischen Motivation benutzt werden.

H4: Politische Motivation hat einen moderierenden Effekt auf die Berziehung zwischen erwartetem Nutzen einer E-Participation Anwendung und der Nutzungsabsicht.

Die in dieser Arbeit untersuchten Wirkungszusammenhänge werden in Abbildung 1 veranschaulicht.

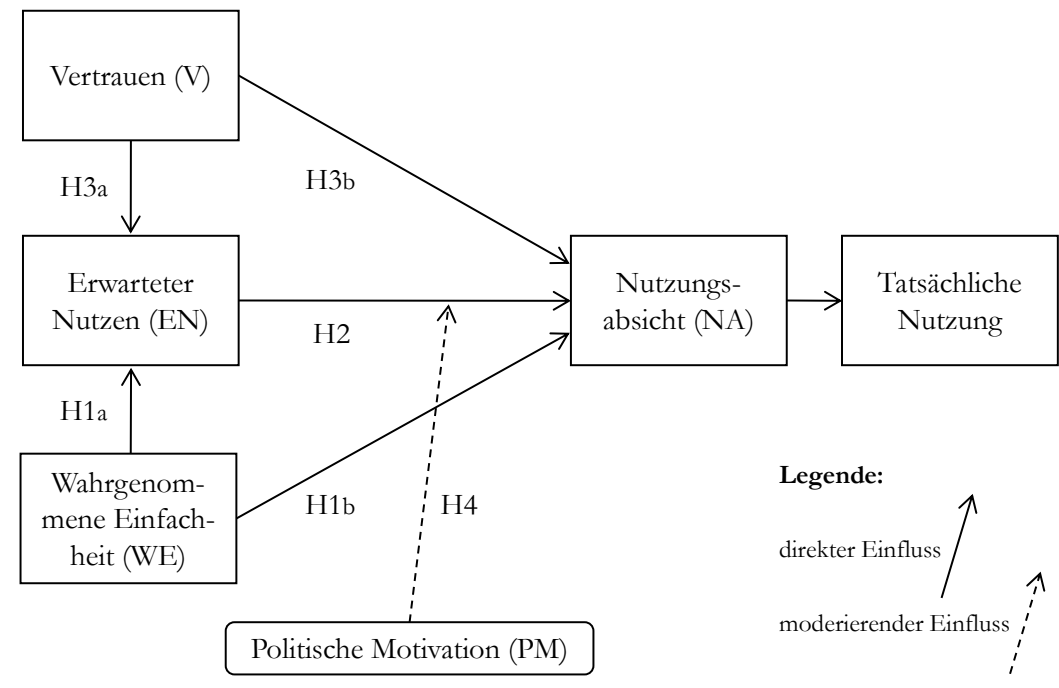

\section{Abbildung 1: Forschungsmodell}

Quelle: Eigene Abbildung

\section{Empirischer Test}

Die Umfrage wurde anhand von zwei E-Participation Anwendungen durchgeführt, die den Teilnehmern zu Beginn des Fragebogens vorgestellt wurden. Dabei handelte es sich um einen politischen Blog, der auf die Webseite von Abgeordneten eingebunden wird, und um das direkte Engagement von Abgeordneten in einer Online-Community. Zu Beginn der Umfrage wurde den Probanden jeweils eine dieser beiden E-Participation Möglichkeiten vorgestellt, auf die sich die anschlieBenden Fragen dann bezogen. Hierzu wurde ein Zufallsmechanismus verwendet.

\subsection{Datenerhebung}

Die verwendeten Fragen für die Identifikation der Konstrukte im Forschungsmodell orientieren sich an Indikatorvariablen, die bereits in anderen Studien erfolgreich eingesetzt wurden. Soweit notwendig wurden die Variablen geringfügig modifiziert, um sie an die Inhalte dieser Studie anzupassen. Tabelle 1 zeigt die Studien, 
die für die Operationalisierung der jeweiligen Konstrukte herangezogen wurden. Dabei wurden jeweils reflektive Messmodelle verwendet, d.h. es wird eine Kausalität unterstellt, die vom untersuchten Konstrukt her auf die Indikatorvariablen zeigt (Chin 1998, S. 35). Die Erfassung der Variablen erfolgte anhand von Aussagen, die auf einer fünfstufigen Likert-Skala zu bewerten waren. Der Fragebogen wurde in einem Pre-Test 15 Probanden zur Beantwortung vorgelegt. Fragen und zu bewertende Aussagen wurden der Kritik entsprechend angepasst.

Die Befragung erfolgte innerhalb von fünf Wochen im Zeitraum vom 09. April bis zum 14. Mai 2009. Hierzu wurden Personen über den Newsletter des Mitglieds des Europäischen Parlaments (MdEP) Herrn Daniel Caspary und über den Bekanntenkreis der Autoren zur Teilnahme an der Umfrage animiert. Die Umfrage wurde zudem auf Twitter, Facebook, wer-kennt-wen, und der Homepage von Herrn Caspary bekannt gemacht. Darüber hinaus wurden alle Studienteilnehmer gebeten, weitere Teilnehmer für die Befragung zu gewinnen, um so eine möglichst hohe Teilnehmerzahl zu erzielen. Insgesamt konnte so ein Rücklauf von 151 Teilnehmern erreicht werden. 69 Probanden beantworteten den Fragebogen zur Nutzung von politischen Blogs. 82 Teilnehmer füllten den Fragebogen zum Engagement von Politikern in Online-Communities aus.

Tabelle 1: Operationalisierung der Konstrukte

\begin{tabular}{|l|l|c|c|}
\hline Konstrukt & Quelle & $\begin{array}{c}\text { Indikator- } \\
\text { variablen }\end{array}$ & $\begin{array}{c}\text { Cronbach's } \\
\text { Alpha }\end{array}$ \\
\hline EN: Erwarteter Nutzen & $\begin{array}{l}\text { Davis 1989; Carter und Bélanger } \\
2005\end{array}$ & 4 & 0,84 \\
\hline $\begin{array}{l}\text { WE: Einfachheit der } \\
\text { Nutzung }\end{array}$ & Wu und Chen 2005; Davis 1989 & 4 & 0,84 \\
\hline V: Vertrauen & $\begin{array}{l}\text { Wu und Chen 2005; Hassanein } \\
\text { und Head 2007 }\end{array}$ & 4 & 0,89 \\
\hline NA: Nutzungsabsicht & Suh 2003 & 3 & 0,94 \\
\hline PM: Politische Motivation & eigene Aufstellung & 3 & 0,91 \\
\hline
\end{tabular}

\subsection{Auswertung}

Das durchschnittliche Alter der Umfrageteilnehmer liegt bei 28 Jahren. Die Teilnehmer gliedern sich in Studenten (41,1\%), Angestellte (29,1\%), Beamte (9,9\%), Selbstständige (8,6\%), Schüler $(6,0 \%)$, nicht Erwerbstätige (3,3\%) und Rentner $(1,3 \%)$. Ein Umfrageteilnehmer $(0,7 \%)$ machte keine Angabe bzgl. seiner Tätigkeit.

Die Reliabilität der Konstruktmessung wurde mit der Kennzahl Cronbach's Alpha überprüft (Cronbach 1951, S. 299). Die Werte liegen im Bereich von 0,84 bis 0,94 und deuten auf eine hohe Konsistenz der Konstruktmessung hin, vgl. Tabelle 1. Zur Auswertung des postulierten Modells wird die varianzerklärende Kausalanalyse nach dem Partial Least Squares (PLS) Ansatz herangezogen, da die- 
ser Ansatz bei Stichprobengrößen von unter 200 Teilnehmern besser geeignet ist als die kovarianzerklärende Variante (Homburg et al. 2008, S. 572). Die Berechnung der entsprechenden Strukturzusammenhänge wurde mit der Software SmartPLS 2 durchgeführt. Die Faktorladung der einzelnen Indikatorvariablen liegt in allen Fällen über dem empfohlenen Wert von 0,7 (Chin 1998, S. xiii). Die einzelnen Werte werden in Tabelle 2 dargestellt.

Die durchschnittlich erklärte Varianz (DEV) beschreibt den gemeinsam erfassten

Tabelle 2: Faktorladungen

\begin{tabular}{|c|c|c|c|c|c|}
\hline $\begin{array}{c}\text { Indika- } \\
\text { torvariab- } \\
\text { le }\end{array}$ & $\begin{array}{l}\text { Faktor- } \\
\text { ladung }\end{array}$ & $\begin{array}{l}\text { Indikator- } \\
\text { variable }\end{array}$ & $\begin{array}{l}\text { Faktor- } \\
\text { ladung }\end{array}$ & $\begin{array}{l}\text { Indikator- } \\
\text { variable }\end{array}$ & $\begin{array}{l}\text { Faktor- } \\
\text { ladung }\end{array}$ \\
\hline $\mathrm{V} 1$ & 0,87 & WE3 & 0,91 & PM1 & 0,92 \\
\hline V2 & 0,80 & WE4 & 0,88 & PM2 & 0,92 \\
\hline V3 & 0,85 & EN1 & 0,73 & PM3 & 0,91 \\
\hline V4 & 0,95 & EN2 & 0,86 & NA1 & 0,94 \\
\hline WE1 & 0,82 & EN3 & 0,77 & NA2 & 0,96 \\
\hline WE2* & $0,54^{*}$ & EN4 & 0,92 & NA3 & 0,94 \\
\hline
\end{tabular}

Anteil der Varianz zwischen einem Konstrukt und seinen Indikatorvariablen. Die Werte liegen in der Studie zwischen 0,68 und 0,90 und sind damit jeweils besser als der empfohlene Mindestwert von 0,5 (Fornell und Larcker 1981, S. 46). Dabei wird in allen Fällen das strenge Fornell-Larcker-Kriterium erfüllt, nachdem die DEV eines Konstruktes größer als jede quadrierte Korrelation desselben mit den anderen Konstrukten sein muss (ebd., S. 46). Dadurch wird sichergestellt, dass die reflektiv operationalisierten Konstrukte tatsächlich eigenständige Einflüsse darstellen. Die entsprechenden Werte werden in Tabelle 3 dargestellt.

Tabelle 3: DEV und quadrierte Korrelationen

\begin{tabular}{|c|c|c|c|c|c|}
\hline & $\mathbf{V}$ & $\mathbf{W E}$ & $\mathbf{E N}$ & $\mathbf{P M}$ & NA \\
\hline $\mathbf{V}$ & $\mathbf{0 , 7 6}$ & & & & \\
\hline $\mathbf{W E}$ & 0,14 & $\mathbf{0 , 7 6}$ & & & \\
\hline EN & 0,27 & 0,19 & $\mathbf{0 , 6 8}$ & & \\
\hline PM & 0,09 & 0,21 & 0,09 & $\mathbf{0 , 8 4}$ & \\
\hline NA & 0,31 & 0,18 & 0,46 & 0,26 & $\mathbf{0 , 9 0}$ \\
\hline
\end{tabular}

Diagonale Werte stellen die DEV des Konstruktes dar, in den übrigen Zellen werden die quadrierten Korrelationen dargestellt.

${ }^{2}$ Verwendet wurde die Version 2.0.M3 (Ringle et al. 2005) 


\section{Diskussion}

Die Ergebnisse der Kausalanalyse unterstützen in allen bis auf zwei Fällen die aufgestellten Hypothesen (vgl. Tabelle 4). Insgesamt kann durch die im Modell postulierten Wirkungszusammenhänge bis zu $60 \%$ der Varianz $\left(\mathrm{R}^{2}\right)$ der Nutzungsabsicht erklärt werden.

Tabelle 4: Hypothesentests

\begin{tabular}{|c|c|c|c|c|}
\hline & Zusammenhang & $\beta$ & $t$-Wert & $\begin{array}{c}\text { Unterstützung der } \\
\text { Hypothese }\end{array}$ \\
\hline $\mathrm{H}_{\mathrm{a}}$ & $\mathrm{WE} \rightarrow \mathrm{EN}$ & 0,28 & $3,59^{* * *}$ & JA \\
\hline $\mathrm{H} 1_{\mathrm{b}}$ & $\mathrm{WE} \rightarrow \mathrm{NA}$ & 0,02 & 0,25 & NEIN \\
\hline $\mathrm{H} 2$ & $\mathrm{EN} \rightarrow \mathrm{NA}$ & 0,47 & $6,77^{* * *}$ & JA \\
\hline $\mathrm{H}_{\mathrm{a}}$ & $\mathrm{V} \rightarrow \mathrm{EN}$ & 0,41 & $5,08^{* * *}$ & JA \\
\hline $\mathrm{H} 3_{\mathrm{b}}$ & $\mathrm{V} \rightarrow \mathrm{NA}$ & 0,21 & $3,08^{* *}$ & JA \\
\hline $\mathrm{H} 4$ & PM moderiert EN $\rightarrow \mathrm{NA}$ & 0,06 & 0,76 & NEIN \\
\hline
\end{tabular}

\subsection{Akzeptanz von E-Participation Anwendungen}

Hypothese $\mathrm{H}_{1}$ a wird unterstützt. Die wahrgenommene Einfachbeit einer E-Participation Anwendung übt einen positiven Einfluss auf den erwarteten Nutzen dieser Anwendung aus. Dieses Ergebnis legt nahe, dass E-Participation Anwendungen so gestaltet werden sollten, dass diese möglichst einfach und intuitiv zu nutzen sind. Denn dadurch steigt die Wahrscheinlichkeit, dass die Anwendung als nützlich angesehen wird.

Hypothese H2 wird ebenfalls unterstützt. Der erwartete Nutzen einer E-Participation Anwendung übt einen substantiell positiven Einfluss auf die Absicht aus, dass diese in der Zukunft genutzt wird. Dieser Zusammenhang stellt dabei den stärksten im Rahmen der Studie gemessenen Einfluss dar. Das Ergebnis unterstreicht die Bedeutung eines klaren Nutzenversprechens für elektronische Beteiligungsmöglichkeiten. Es empfiehlt sich daher E-Participation Anwendungen so zu gestalten und zu bewerben, dass der Nutzen, d. h. die Möglichkeit zur politischen Beteiligung, klar erkennbar ist.

Die beiden Hypothesen $\mathrm{H}_{3}$ und $\mathrm{H}_{3}$ berden ebenfalls unterstützt. Vertrauen in eine E-Participation Anwendung übt (a) einen substantiell positiven Einfluss auf den erwarteten Nutzen und (b) einen schwachen positiven Einfluss auf die Nutzungsabsicht aus. Diese Ergebnisse legen nahe, dass E-Participation Anwendungen bereits bei der Entwicklung so gestaltet werden sollten, dass Sicherheit und Datenschutz ein hoher Stellenwert eingeräumt wird. 


\subsection{Nicht signifikante Ergebnisse}

Unerwartete Ergebnisse sind ebenfalls interessant $\mathrm{zu}$ bewerten. Hypothese $\mathrm{H} 1_{\mathrm{b}}$ wird nicht unterstützt. Es lässt sich kein signifikanter direkter Einfluss der wahrgenommenen Einfachheit auf die Nutzungsabsicht nachweisen. Dieses Ergebnis ist durchaus konsistent zu früheren Studien der Akzeptanz von Technologien, in denen dieser direkte Effekt zwar in mehreren Fällen signifikant war, aber jeweils eine deutlich schwächere Korrelation zur Nutzungsabsicht aufwies, als zum erwarteten Nutzen (Davis 1989, S. 333; Davis et al. 1989, S. 997; Adams et al. 1992, S. 237, 241; Venkatesh und Davis 2000, S. 197).

Hypothese H4 wird ebenfalls nicht unterstützt. Es lässt sich ein signifikanter direkter Einfluss von politischer Motivation auf die Nutzungsabsicht zeigen $(\beta=0,32 ; \mathrm{p}<0,001)$. Das bedeutet, dass politisch motivierte Bürger eher dazu neigen eine E-Participation Anwendung zu nutzen, als politisch weniger Interessierte. Allerdings kann kein moderierender Einfluss auf die Beziehung zwischen erwartetem Nutzen und Nutzungsabsicht nachgewiesen werden. Dieses Ergebnis legt nahe, dass politisch motivierte Bürger, entgegen unserer Annahme, nicht überproportional stark durch einen hohen erwarteten Nutzen für die Verwendung einer E-Participation Anwendung begeistert werden können. Anders ausgedrückt bedeutet dies, dass bei der Steigerung des Nutzens einer E-Participation Anwendung sowohl politisch motivierte, als auch politisch weniger interessierte Bürger, in etwa gleichen Anteilen hinzugewonnen werden können.

Gerade dieses letzte Ergebnis erscheint von besonderer Bedeutung, da es eine Möglichkeit aufzeigt, wie neben bereits politisch motivierten Bürgern auch weniger interessierte Bürger für eine Beteiligung am politischen Meinungsbildungsprozess begeistert werden könnten.

\subsection{Einschränkungen der Studie}

Die vorliegende Studie unterliegt einer Reihe von Einschränkungen, die hervorgehoben werden sollten. Zunächst wurde in der Befragung nur die Absicht einer Nutzung und nicht die tatsächliche Nutzung gemessen. Nach der Theory of Reasoned Action (Fishbein und Ajzen 1975, S. 381) kann jedoch eine starke Korrelation zwischen der Absicht und der tatsächlichen Nutzung angenommen werden. Dieser Zusammenhang konnte ebenfalls in Bezug auf TAM bestätigt werden (Bernadette 1996, S. 90).

In früheren Studien zur Akzeptanz von Technologien wurden häufig Studenten als Probanden herangezogen, was die Repräsentativität der Stichprobe entsprechend einschränkt (Davis et al. 1989, S. 983; Adams et al 1992; Taylor und Todd 1995, S. 144). Auch in dieser Studie wurden Studenten für die Umfrage gewonnen, allerdings waren diese lediglich zu 41\% unter den Umfrageteilnehmern vertreten. Damit liegt der Anteil an Studenten zwar unter dem von anderen Studien, doch nach wie vor sind diese damit überdurchschnittlich stark in der Umfrage repräsen- 
tiert. Entsprechend verdeutlicht auch das Durchschnittsalter von 28 Jahren, dass in dieser Studie tendenziell ein jüngeres Publikum befragt wurde.

Einschränkend ist ebenfalls hervorzuheben, dass die Umfrageteilnehmer über den Bekanntenkreis der Autoren hinaus, ebenfalls über den Newsletter des MdEP Herrn Daniel Caspary und über Einträge in den Online-Communities Twitter, Facebook und wer-kennt-wen gewonnen wurden. Daher kann davon ausgegangen werden, dass die Teilnehmer sowohl technisch als auch politisch eine eher überdurchschnittlich interessierte Zielgruppe darstellen.

\section{Zusammenfassung und Ausblick}

Die Ergebnisse dieser Arbeit bestätigen bisherige Untersuchungen zur Akzeptanz von Technologien und zeigen, dass diese auch in Bezug auf E-Participation Anwendungen gültig sind. Insbesondere wird die Bedeutung eines klaren Nutzenversprechens und einer vertrauenswürdigen Gestaltung elektronischer Beteiligungsmöglichkeiten hervorgehoben. Die Möglichkeit einer einfachen Nutzung von E-Participation Anwendungen konnte als indirekter Einflussfaktor identifiziert werden, der sich positiv auf den erwarteten Nutzen auswirkt. Darüber hinaus weisen die Ergebnisse darauf hin, dass durch die Steigerung des Nutzens einer Anwendung sowohl politisch motivierte als auch politisch weniger interessierte Bürger für die Beteiligung am politischen Willensbildungsprozess hinzugewonnen werden können. Dadurch wird die Bedeutung eines klaren Nutzenversprechens erneut unterstrichen. Dabei bietet gerade die Möglichkeit, auch politisch weniger interessierte Bürger für eine Beteiligung zu gewinnen, einen interessanten Ansatzpunkt für weitere Forschungen.

\section{Literatur}

Adams, D, Nelson, R, Todd, P (1992) Perceived usefulness, ease of use, and usage of information technology: a replication. MIS Quarterly 16(2):227-247.

Albrecht, S, Kohlrausch, N, Kubicek, H (2008) E-Partizipation - Elektronische Beteiligung von Bevölkerung und Wirtschaft am E-Government. Studie im Auftrag des Bundesministeriums des Innern, Ref. IT 1, Bremen.

Bernadette, S (1996) Empirical evaluation of the revised technology acceptance model. Management Science 42(1):85-93.

Bräuer, M, Seifert, M, Wolling J (2008) Politische Kommunikation 2.0 Grundlagen und empirische Ergebnisse zur Nutzung neuer Partizipationsformen im Internet. In: Zerfaß A (Hrsg) Kommunikation, Partizipation und Wirkungen im Social Web: Strategien und Anwendungen, Perspektiven für Wirtschaft, Politik und Publizistik. Halem, Köln. 
Caddy, J, Vergez, C (2001) Citizens as partners: Information, consultation and public participation in policy-making. OECD Publishing.

Carter, L, Bélanger, F (2005) The utilization of e-government services: citizen trust, innovation and acceptance factors. Information Systems Journal 15:15-25.

Chin, W (1998) Issues and opinion on structural equation modeling. MIS Quarterly 22(I): vii-xvi.

Coleman, S, Gotze, J, (2001) Bowling together: Online public engagement in policy deliberation. Hansard, London.

Cronbach, LJ (1951) Coefficient alpha and the internal structure of tests, Psychometrika 16(3):297-333.

Davis, FD (1989) Perceived usefulness, perceived ease of use, and user acceptance of information technology. MIS Quarterly 13(3):318-339.

Davis, FD, Bagozzi, RP, Warshaw, PR (1989) User acceptance of computer technology: a comparison of two theoretical models. Management Science 35(8):982-1003.

Dutton, WH, Shepherd, A (2006) Trust in the internet as an experience technology. Information, Communication \& Society 9(4):433-451.

Feldmer, S (2008) Bloggen statt Ortsverein. Süddeutsche Zeitung, Ausgabe vom 19. April 2008.

Fishbein, M, Ajzen, I (1975) Belief, attitude, intention, and behavior: an introduction to theory and research. Addison-Wesley.

Fornell, C, Larcker, D (1981) Evaluating structural equation models with unobservable variables and measurement errors, Journal of Marketing Research, 18(1):39-50.

Friedmann, B, Kahn, P, Howe, D (2000) Trust online. Communications of the ACM 43(12):34-40.

Hassanein, K, Head, M (2007) Manipulating perceived social presence through the web interface and ist impact on attitdue towards online shopping. International Journal of Human Computer Studies 65:689-708.

Homburg, C, Pflesser C, Klarmann, M (2008) Strukturgleichungsmodelle mit latenten Variablen: Kausalanalyse. In: Herrmann, A, Homburg, C, Klarmann, M (Hrsg) Handbuch Marktforschung. 3. Auflage, Gabler, Wiesbaden.

Jansen, G, Dowe, C, Heimann, U (2006) Facilitating active citizenship:

E-Participation in Grossbritannien \& Deutschland - Eine Bestandsaufnahme. http://www.britishcouncil.de/e/society/e_participation.htm.

Abruf am 2009-09-16.

Jeyaraj, A, Rottman, JW, Lacity, MC (2006) A review of the predictors, linkages, and biases in IT innovation adoption research. Journal of Information Technology 21(1):1-23. 
Kubicek, H, Westholm, H (2005) Scenarios for future use of e-democracy tools in Europe. International Journal of Electronic Government Research 1(3):33-50.

Lucke, J, Reinermann, H (2000) Speyerer Defintion von Electronic Government. http://foev.dhv-speyer.de/ruvii/SP-EGov.pdf. Abruf am 2009-09-16.

Macintosh, A (2008) E-Democracy and E-Participation research in Europe. Digital Government 17.

Moorstedt, T (2009) Ich bin es, Dein Anführer. Süddeutsche Zeitung, Ausgabe vom 18. Februar 2009.

O'Reilly, T (2005) What is web 2.0? Design patterns and business models for the next generation of software. http://www.oreillynet.com/pub/a/oreilly/tim/ news/2005/09/30/what-is-web-20.html. Abruf am 2009-09-16.

Parvez, Z (2008) E-Democracy from the perspective of local elected members. International Journal of Electronic Government Research 4(3):20-33.

Pavlou, PA (2003) Consumer acceptance of electronic commerce-integrating trust and risk with the technology acceptance model. International Journal of Electronic Commerce 7(3):69-103.

Ringle, CM, Wende, S, Will, S (2005) SmartPLS 2.0 (M3) Beta, Hamburg http://www.smartpls.de. Abruf am 2009-09-16.

Sclove, R (1995) Democracy and technology. Guilford, New York.

Stern, J (2008) Web 2.0 trifft Politik 3.11: Bringt politische Kommunikation durch das Internet mehr Transparenz, Partizipation und Legitimität?. In: Patzelt, W (Hrsg) Res publica semper reformanda. Verlag für Sozialwissenschaften, Wiesbaden.

Suh, B (2003) The impact of customer trust and perception of security control on the acceptance of electronic commerce. International Journal of Electronic Commerce 7(3):135-161.

Sun, H, Zhang, P (2006) The role of moderating factors in user technology acceptance. International Journal of Human-Computer Studies 64:53-78.

Taylor, S, Todd, PA (1995) Understanding information technology usage: a test of competing models. Information Systems Research 6(4):144-176.

Venkatesh, V, Davis, FD (2000) A theoretical extension of the technology acceptance model: four longitudinal field studies. Management Science 46(2):186-204.

Venkatesh, V, Morris, M, Davis, FD (2003) User acceptance of information technology: toward a unified view. Management Information Quarterly 27(3):425-478. 
Westholm, H, Wimmer, M (2007) Interdisciplinary framework to address the sociotechnical and political challenges of E-Participation. http://www.demonet.org/what-is-it-about/research-papers-reports-1/demo-netdeliverables/AichholzerEtAl2007b. Abruf am 2009-09-16.

Wu, IL, Chen, JL (2005) An extension of trust and TAM model with TPB in the initial adoption of on-line tax: an empirical study. International Journal of Human-Computer Studies 62:784-808. 